
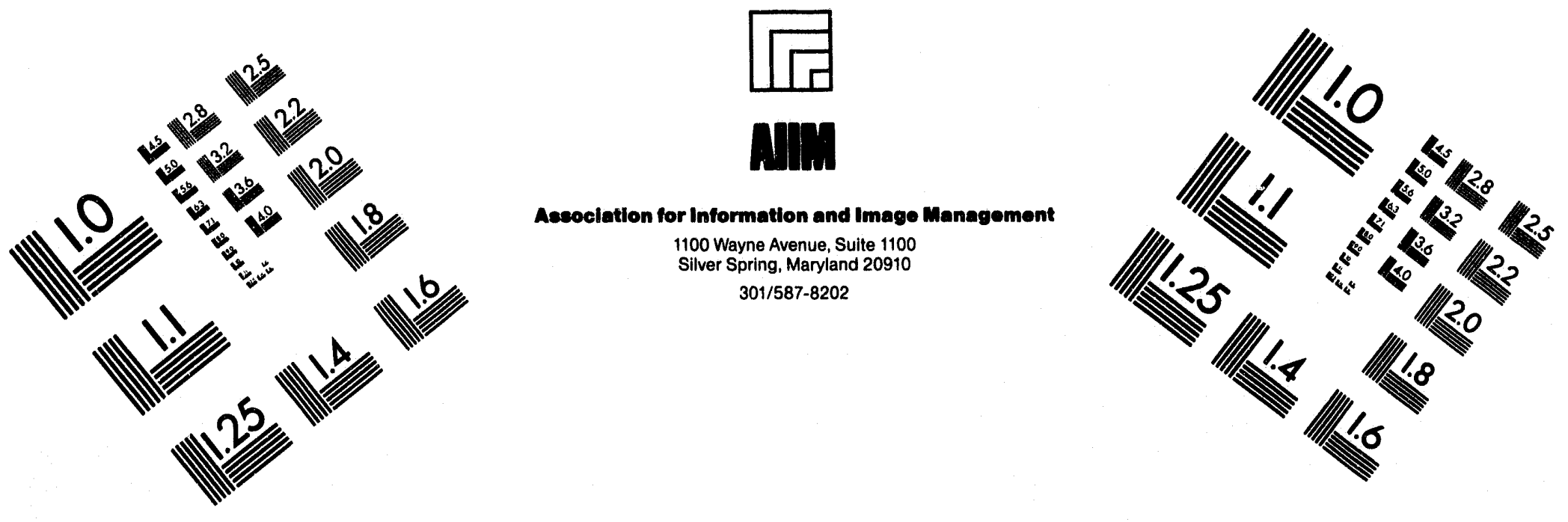

\title{
Centimeter
}

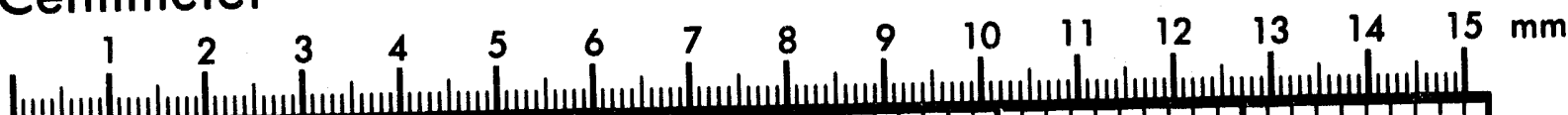

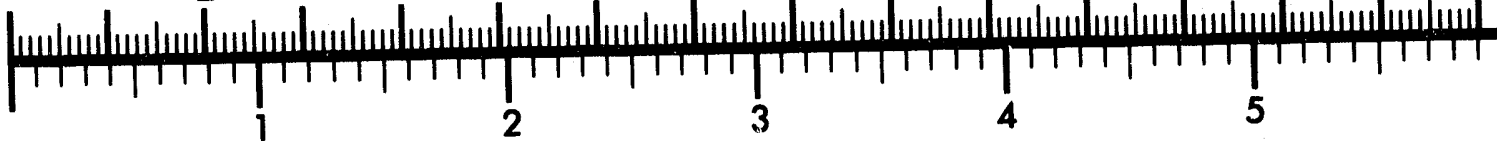
Inches
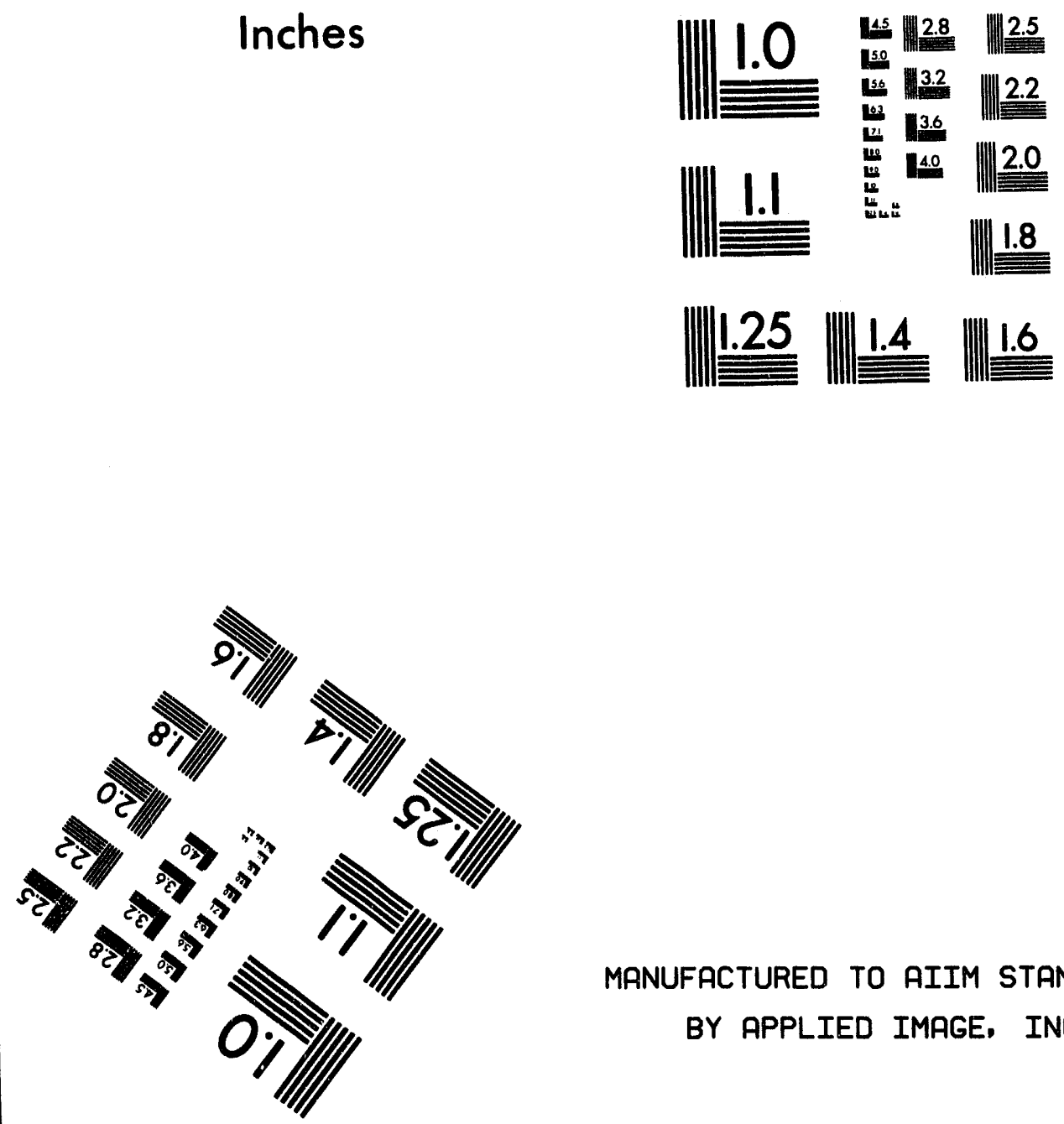

MANUFACTURED TO AIIM STANDARDS

BY APPLIED IMAGE, INC.

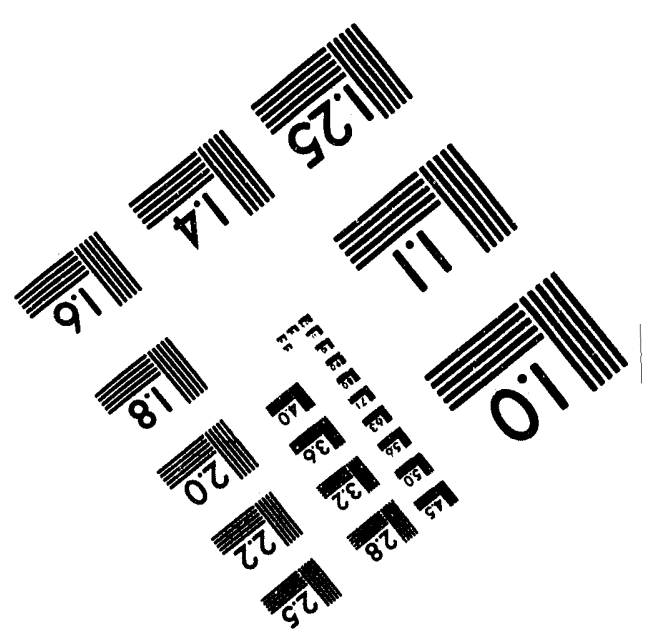





\title{
ANK/TD/CP.-81662
Conf $-940664=-14$
}

Paper

\section{MHD FLOW IN RECTANGULAR DUCTS WITH INCLINED NON-UNIFORM TRANSVERSE MAGNETIC FIELD*}

by

\author{
THANH Q. HUA \\ Fusion Power Program \\ Technology Development Division \\ Argonne National Laboratory \\ Argonne, IL 60439 USA \\ JOHN S. WALKER \\ Department of Mechanical and Industrial Engineering \\ University of Illinois \\ Urbana, IL 61801 USA
}

\begin{abstract}
The wubmitted menuscript has been suthored
by contrector of the U.S. Government

under contrect No. W.31-108-ENG-38.

Accordingly, the U.S. Government retains a

Accordingly, the U. S. Govers licones to publich

nonexcluaive, royalry-lree lleona to publlan

or reproduce the publisted form of this

contribution, or sllow others to do $\infty 0$, for

U. S. Government purpoese.
\end{abstract}

June 1994

Submitted to the Third International Symposium on Fusion Nuclear Technology, University of California at Los Angeles, June 27 - July 1, 1994.

* Work supported by the United States Department of Energy/Office of Fusion Energy, under Contract No. W-31-109-Eng-38.

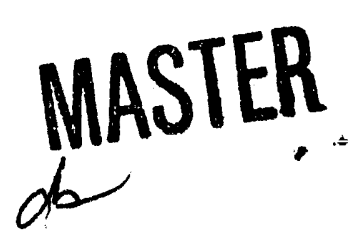




\title{
MHD FLOW IN RECTANGULAR DUCTS WITH INCLINED NON- UNIFORM TRANSVERSE MAGNETIC FIELD*
}

Thanh Q. Hua

Fusion Power Program

Technology Development Division

Argonne National Laboratory

9700 South Cass Avenue

Argonne, IL 60439 USA
John S. Walker

Department of Mechanical and

Industrial Engineering

University of Illinois

Urbana, IL 61801 USA

\begin{abstract}
This paper examines the three-dimensional liquid metal MHD flow in rectangular ducts with thin conducting walls and with an inclined nonuniform transverse magnetic field. The Hartmann number and interaction parameter are assumed to be large and the magnetic Reynolds number is assumed to be small. Under these assumptions, viscous and inertial effects are confined to thin boundary layers adjacent to the walls. Outside these layers, the governing equations are significantly simplified.
\end{abstract}

For validation of the numerical solutions, exact analytical solutions are derived for the case of a rectangular duct of equal wall thickness and with a uniform magnetic field. Comparisons of the exact analytical and numerical solutions give excellent agreement. Variation of the fully developed flow pressure gradient with the wall conductance ratio, aspect ratio, and magnetic angle is discussed. Numerical solutions are presented for flow in the varying field region where the flow is perturbed due to three-dimensional effects. The three-dimensional pressure drop, i.e., in excess of the locally fully developed pressure, is presented and its implication to a fusion blanket is discussed. The velocity distributions are also presented.

* Work supported by the U.S. Department of Energy, Office of Fusion Energy, under Contract No. W-31-109-Eng-38.

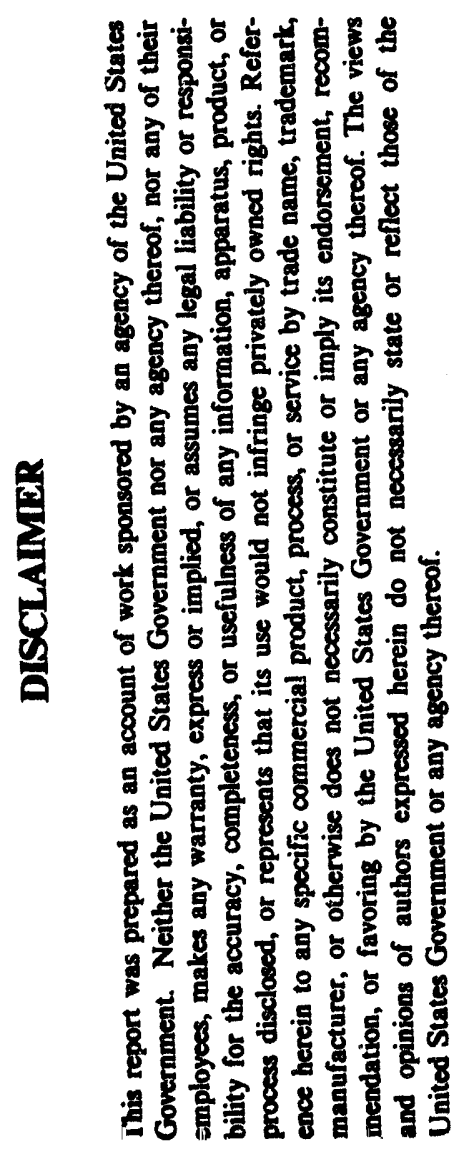




\section{Introduction}

In a liquid metal self-cooled tokamak blanket, the liquid metal, typically $\mathrm{Li}$ or $\mathrm{LiPb}$ serves both functions as coolant and as tritium breeding materials. A simple poloidal blanket has multiple arrays of rectangular poloidal coolant channels which are connected to inlet and outlet manifolds. The manifolds are located at the top and/or bottom of the machine. The blanket consists of several modules, typically greater than 20, to provide complete toroidal coverage. Because of the modular construction, the toroidal field lines intersect the side walls of the coolant channels at a small angle of a few degrees.

In previous three-dimensional MHD analyses ("core flow approach") of liquid metal flow in rectangular duct with conducting walls and with nonuniform magnetic field [1-3], the field lines are treated as parallel to a pair of walls (side walls) and perpendicular to a pair of Hartmann walls. Analysis has been carried out for ducts with arbitrary cross section and nearly arbitrary orientation relative to the field [4]. In a rectangular duct where the side walls are parallel to the field lines, the side layers adjacent to the side carry a significant fraction of the total flow in the form of high velocity jets. For instance, under strong uniform magnetic field the side layers of a square duct with thin conducting wall carry about $25 \%$ of the total flow. Outside these layers, i.e. in the core, the velocity is uniform. The core flow determines the fully developed pressure gradient while the side layer flow "rides" free. Some liquid metal blanket concepts have assumed perfect alignment of the toroidal field with the first wall coolant channels to take advantage of the favorable heat transfer characteristics of the side layers. However, heat transfer analysis for fully developed 
laminar flow with an inclined magnetic field [5] revealed substantial changes of the wall temperature for magnetic angle of only a few degrees.

In this paper we examine the flow of liquid metal in thin conducting rectangular ducts with an inclined non-uniform transverse magnetic fields. The field lines intersect the side walls at an angle $\theta$. As $\theta$ deviates from zero the side layers begin to detach from the side walls and the flow comes out of the side layers and into the core. As a result, the core velocity is not uniform and the pressure gradient increases. The scope of this analysis is confined to an angle $\theta$ which is sufficiently large (no less than a few degrees) so that complete detachment of the side layers from the side walls can be assumed. Therefore, the boundary layers at all walls are Hartmann layers which carry no significant flow and which have well known local exponential solution structure. This analysis seeks the solutions of the three-dimensional core variables that match the Hartmann solutions at the core/Hartmann layer interface. For fully developed flow in uniform magnetic field analytical solutions are derived and used to validate the numerical solutions.

\section{Governing Equations and Boundary Conditions}

We consider the steady flow of an incompressible liquid metal in a rectangular duct with thin conducting walls and with an externally applied transverse magnetic field whose strength varies along the duct. The magnetic field lines intersect the pair of side walls at an angle $\theta$ (Fig. 1). The much weaker axial and induced magnetic fields are neglected in this model because the major body force in the liquid metal arises from the interaction between the fluid flow and the strong transverse field. Two 
important dimensionless parameters in any MHD problem are the Hartmann number $\mathrm{M}$ and interaction parameter $\mathrm{N}$ defined by

$$
\begin{aligned}
& M=B_{0} L\left(\frac{\sigma}{\rho v}\right)^{1 / 2} \\
& N=\frac{\sigma B_{0}^{2} L}{\rho U_{0}}
\end{aligned}
$$

where $\sigma, \rho, v$ are the fluid electrical conductivity, density, and kinematic viscosity respectively, $\mathrm{U}_{0}$ is the average axial velocity, $\mathrm{L}$ is a characteristic length, and $B_{0}$ is the characteristic magnetic flux density. In fusion blankets, the values of $\mathrm{M}$ and $\mathrm{N}$ are very large, in the order of $10^{3}$ to $10^{5}$. This means that the viscous and inertial effects are negligible and the electromagnetic force is dominant in determining the flow and pressure distributions except in thin boundary and possibly shear layers.

In previous analysis with $\theta=0^{\circ}$ [1], the flow in the side layers, $Q_{s}$, is related to the total flow (core plus side layers), $Q_{\mathfrak{t}}$, by

$$
\frac{Q_{s}}{Q_{t}}=\left[1+\frac{3 c_{s}}{a c_{h}}+\frac{3 c_{s}}{a^{2}}\right]^{-1}
$$

where $a$ is the duct aspect ratio, $c_{h}$ and $c_{s}$ are the wall conductance ratios of the Hartmann wall and side wall, respectively. The wall conductance ratio is

$$
c=\frac{\sigma_{w} t}{\sigma L}
$$


where $\sigma_{w}$ and $t$ are the electrical conductivity and thickness of the wall. The analysis assumes that $c_{s}$ is much greater than the $0\left(a^{1 / 2} M^{-1 / 2}\right)$ side layer thickness. With this assumption details of the side layer can be ignored provided that the boundary value problem for the side layer is well posed. An improved approach treats $c_{s}$ the same order as the side layer thickness with a composite of the core and side layer solutions [3]. In either approach, the flow in the side layer is distributed parabolically along the side wall with zero flow at the two corners.

For $\theta>0^{\circ}$, the side layers are detached from the side walls and the flow in the side layers enter the core. The present analysis treats cases where complete detachment of the side layers can be assumed, i.e., the angle $\theta$ exceeds a lower bound value. This minimum angle, $\theta_{\min }$, is determined from the intersection of the side layer (when $\theta=0^{\circ}$ ) with the shear layer along the $y$ axis at $\mathrm{z}=0$ (Fig. 1) such that the overlap length at the bottom, $d$, is relatively small

$$
\theta_{\min }=\tan ^{-1}\left(\frac{\mathrm{a}^{1 / 2} \mathrm{M}^{-1 / 2}}{\mathrm{~d}}\right)
$$

For $d=0.1, a=1 ., M=5 . e 4$, then $\theta_{\min }=2.6^{\circ}$ which is likely smaller than the intersection angle of the toroidal field with the blanket modules.

The dimensionless equations governing the inviscid and inertialess flow of the liquid metal are

$$
\underset{\sim}{\nabla P}=\underset{\sim}{\mathrm{j}} \times \underset{\sim}{\mathrm{B},} \quad \underset{\sim}{\mathrm{j}}=-\underset{\sim}{\nabla} \phi+\underset{\sim}{\mathrm{v}} \times \mathrm{B},
$$

and

$$
\underset{\sim}{\nabla \cdot v=0,} \quad \underset{\sim}{\sim} \cdot \mathrm{j}=0 .
$$


Here $p, j, v$, and $\phi$ are the pressure, electric current density, velocity, and electric potential, normalized by $\sigma \mathrm{U}_{0} \mathrm{~B}_{0}^{2} \mathrm{~L}, \sigma \mathrm{U}_{0} \mathrm{~B}_{0}, \mathrm{U}_{0}$, and $\mathrm{U}_{0} \mathrm{~B}_{0} \mathrm{~L}$, respectively. Outside the boundary layers the core components of velocity and current density are

$$
\begin{aligned}
& u_{c}(x, y, z)=\beta \frac{\partial \phi_{c}}{\partial z}-\beta^{2} \frac{\partial p}{\partial x} \\
& w_{c}(x, y, z)=-\beta \frac{\partial \phi_{c}}{\partial x}-\beta^{2} \frac{\partial p}{\partial z}, \\
& \frac{\partial v_{c}}{\partial y}=-\frac{\partial u_{c}}{\partial x}-\frac{\partial w_{c}}{\partial z}, \\
& j_{x c}(x, y, z)=\beta \frac{\partial p}{\partial z}, \\
& j_{z c}(x, y, z)=-\beta \frac{\partial p}{\partial x}, \\
& \frac{\partial j_{y}}{\partial y}=-\beta^{\prime} \frac{\partial p}{\partial z} .
\end{aligned}
$$

Where $p(x, z)$ is the pressure, which is constant along the magnetic field lines, $\beta(x)=1 / B_{y}(x)$ and $\beta^{\prime}(x)=d \beta / d x$. The electric potential in the core along the magnetic field lines is obtained from Eqs. (1b) and (2f).

$$
\phi_{c}(x, y, z)=\frac{1}{2}\left[y^{2}-y\left(f_{t}+f_{b}\right)+f_{t} f_{b}\right] \beta^{\prime} \frac{\partial p}{\partial z}+\frac{y-f_{b}}{f_{t}-f_{b}} \phi_{t}+\frac{f_{t}-y}{f_{t}-f_{b}} \phi_{b}
$$


here the subscripts "t" and " $b$ " denote variables at the top and bottom walls whose coordinates are defined by the functions $f_{t}$ and $f_{b}$ (see Fig. 1).

The boundary conditions for the core variables are [6]

$$
\underset{\sim}{\mathrm{v}_{\mathrm{c}}} \cdot \hat{\mathrm{n}}=0 \quad, \quad \underset{\sim}{\mathrm{j}_{\mathbf{c}}} \cdot \hat{\mathrm{n}}=\mathrm{c} \nabla^{2} \phi_{\mathrm{w}}
$$

where $\hat{\mathbf{n}}$ is a unit normal to the wall into the liquid metal. Conditions $(3 a, b)$ neglect the $0\left(\mathrm{M}^{-1}\right)$ jumps in $\mathrm{v}, \mathrm{j}$, and $\phi$ across the Hartmann layer, which has a local exponential structure and which matches any tangential velocity in the adjacent inviscid core region and satisfies the no-slip conditions at the wall. Condition ( $3 \mathrm{~b})$, also known as the thin conducting wall condition, implies $t / L \ll 1$ and the electric potential at the wall is uniform through its thickness.

\section{Reduced Governing Equations and Boundary Conditions}

The three dimensional problem in the core can be completely solved once the solutions for the variables $\mathrm{p}(\mathrm{x}, \mathrm{z}), \phi_{\mathrm{t}}(\mathrm{x}, \mathrm{z})$, and $\phi_{\mathrm{b}}(\mathrm{x}, \mathrm{z})$ are obtained. The equations governing these two-dimensional variables can be derived by applying boundary conditions $(3 a, b)$ to the core variables.

We integrate Eq. (2c) from $y=f_{b}$ to $y=f_{t}$ and apply boundary condition (3a) at the walls to arrive at an equation in $p(x, z)$

$$
\begin{aligned}
\left(f_{t}-f_{b}\right) \frac{\partial}{\partial x}\left(\beta^{2} \frac{\partial p}{\partial x}\right)+\frac{\partial}{\partial z}\left\{\left[\left(f_{t}-f_{b}\right) \beta^{2}+\frac{1}{12} \beta^{\prime 2}\left(f_{t}-f_{b}\right)^{3}\right] \frac{\partial p}{\partial z}\right\} \\
=\frac{1}{2} \beta^{\prime} \frac{\partial}{\partial z}\left[\left(f_{t}-f_{b}\right)\left(\phi_{t}+\phi_{b}\right)\right]+f_{b}^{\prime} \frac{\partial}{\partial x}\left(\beta \phi_{b}\right)-f_{t}^{\prime} \frac{\partial}{\partial x}\left(\beta \phi_{t}\right)
\end{aligned}
$$


where $f_{t}^{\prime}=d f_{t} / d z$ and $f_{b}^{\prime}=d f_{b} / d z$.

The equations governing the top and bottom wall potential are obtained by applying the boundary condition ( $3 b$ ) and using Eqs. (2e,f)

$$
\begin{aligned}
& c_{n}\left[\frac{\partial^{2} \phi_{t}}{\partial x^{2}}+\cos ^{2} \theta \frac{\partial^{2} \phi_{t}}{\partial z^{2}}\right]=-\sin \theta \beta \frac{\partial p}{\partial x}+\frac{\cos \theta}{2}\left(f_{t}-f_{b}\right) \beta^{\prime} \frac{\partial p}{\partial z}+\cos \theta \frac{\phi_{t}-\phi_{b}}{f_{t}-f_{b}} \\
& c_{e}\left[\frac{\partial^{2} \phi_{t}}{\partial x^{2}}+\sin ^{2} \theta \frac{\partial^{2} \phi_{t}}{\partial z^{2}}\right]=\cos \theta \beta \frac{\partial p}{\partial x}+\frac{\sin \theta}{2}\left(f_{t}-f_{b}\right) \beta^{\prime} \frac{\partial p}{\partial z}+\sin \theta \frac{\phi_{t}-\phi_{b}}{f_{t}-f_{b}} \\
& c_{w}\left[\frac{\partial^{2} \phi_{b}}{\partial x^{2}}+\sin ^{2} \theta \frac{\partial^{2} \phi_{b}}{\partial z^{2}}\right]=-\cos \theta \beta \frac{\partial p}{\partial x}+\frac{\sin \theta}{2}\left(f_{t}-f_{b}\right) \beta^{\prime} \frac{\partial p}{\partial z}-\sin \theta \frac{\phi_{t}-\phi_{b}}{f_{t}-f_{b}} \\
& c_{s}\left[\frac{\partial^{2} \phi_{b}}{\partial x^{2}}+\cos ^{2} \theta \frac{\partial^{2} \phi_{b}}{\partial z^{2}}\right]=\sin \theta \beta \frac{\partial p}{\partial x}+\frac{\cos \theta}{2}\left(f_{t}-f_{b}\right) \beta^{\prime} \frac{\partial p}{\partial z}-\cos \theta \frac{\phi_{t}-\phi_{b}}{f_{t}-f_{b}} .
\end{aligned}
$$

The boundary conditions of Eqs. $(4,5)$ are derived as follows. Sufficiently upstream $\left(x_{u}\right)$ and downstream $\left(x_{d}\right)$ of the varying field region, the flow is fully developed, so there are no axial currents in the fluid or in the wall. The appropriate boundary conditions are

$$
\begin{array}{ll}
p=p_{1} & \text { at } x=x_{u} \\
p=p_{2} & \text { at } x=x_{d}
\end{array}
$$

and

$$
\frac{\partial \phi_{\mathrm{t}}}{\partial \mathrm{x}}=\frac{\partial \phi_{\mathrm{b}}}{\partial \mathrm{x}}=0 \quad \text { at } \mathrm{x}=\mathrm{x}_{\mathrm{u}}, \mathrm{x}_{\mathrm{d}}
$$


The constants $p_{1}$ and $p_{2}\left(p_{1}>p_{2}\right)$ are arbitrarily chosen. After the solution is found, every variable is multiplied by a scaling factor to satisfy the total volumetric flow condition

$$
\int_{z_{w}}^{z_{e}} \int_{b}^{f_{t}} u_{c} d y d z=4 a .
$$

The small sections of the wall corners at the top $\left(z=z_{w}, z_{e}\right)$ are too small to carry any significant axial electric currents, so the axial currents in the fluid adjacent to the corners are also negligible. Therefore

$$
\frac{\partial p}{\partial z}=0 \quad \text { at } z=z_{w}, z_{e}
$$

Condition (9) can also be derived by taking the axial derivative of Eq. (8) and setting it to zero. Finally, continuity of electric potential and current at the wall corners must be satisfied.

\section{Analytical Solutions for Fully Developed Flows}

For uniform magnetic field only, Eqs. (4,5) are substantially simplified with $\frac{\partial}{\partial x}=0, \beta=1$, and $\beta^{\prime}=0$. We further choose a uniform wall conductance ratio, $\mathrm{c}$, and only solve for half the duct, assuming symmetry about $\mathrm{z}_{\mathrm{m}}=\cos \theta-\mathrm{a} * \sin \theta$. The analytical fully-developed flow solutions to the one-dimensional version of Eqs. $(4,5)$ are

- For $-2 a \sin \theta \leq z<0$ : 
$\phi_{\mathrm{t}}(\mathrm{z})=\frac{1}{\sin \theta+\cos \theta}\left[\left(\frac{\tilde{z} \mathrm{P}}{\sin \theta \cos \theta}\right)\left(\frac{\tilde{\mathrm{z}}}{2 \mathrm{c}}+\frac{\cos ^{3} \theta-\sin ^{3} \theta}{\cos ^{2} \theta+\sin \theta \cos \theta}\right)+A_{1} \sin \theta \tilde{\mathrm{z}}^{1 / 2} \mathrm{I}_{1}\left(\mathrm{z}^{*}\right)\right]$

$\phi_{b}(z)=\frac{1}{\sin \theta+\cos \theta}\left[\left(\frac{\tilde{z} P}{\sin \theta \cos \theta}\right)\left(\frac{\tilde{z}}{2 c}-\frac{\cos ^{3} \theta-\sin ^{3} \theta}{\sin ^{2} \theta+\sin \theta \cos \theta}\right)-A_{1} \cos \theta \tilde{z}^{1 / 2} I_{1}\left(z^{*}\right)\right]$

- For $0 \leq \mathrm{z} \leq \mathrm{z}_{\mathrm{m}}$ :

$\phi_{t}(z)=\frac{a P}{2 \cos ^{2} \theta}\left(\frac{z}{c}-\sin \theta\right)+\frac{A_{2}}{2}+\frac{A_{3}}{2} \cosh \left[\left(\frac{1}{a c}\right)^{1 / 2}\left(z-z_{m}\right)\right]$

$\phi_{b}(z)=\frac{a P}{2 \cos ^{2} \theta}\left(\frac{z}{c}+\sin \theta\right)+\frac{A_{2}}{2}-\frac{A_{3}}{2} \cosh \left[\left(\frac{1}{a c}\right)^{1 / 2}\left(z-z_{m}\right)\right]$

where

$$
\begin{aligned}
& \tilde{z}=z+2 a \sin \theta, \\
& z^{*}=2\left[\tilde{z} \frac{(\sin \theta+\cos \theta)}{c}\right]^{1 / 2},
\end{aligned}
$$

$P$ is the fully developed pressure gradient, and $I_{1}\left(z^{*}\right)$ is the modified Bessel function of the first kind and first order. The lengthy expressions for the constants $A_{1}, A_{2}$, and $A_{3}$ are omitted here.

In the absence of experimental data, the exact analytical solutions in Eqs. (10) provide a means to validate the numerical results for fully developed flow conditions. Numerical solutions of Eqs. (4-5) are obtained using $\beta=1$ and $\beta^{\prime}=0$ everywhere in the computational domain and a wide range of the wall conductance ratio, duct aspect ratio, and magnetic angle. 
Comparisons of the wall electric potential are shown in Figs. $2-3$ for $c=0.01$ and $\theta=10^{\circ}$. For clarity of presentation, the computed solutions in the $x y z$ coordinates are transformed and plotted in the $x y^{\prime} z^{\prime}$ orthogonal coordinates normal to the walls. Similarly good agreement between numerical results and analytical solutions was seen for other combinations of $c$ and $\theta$.

Qualitative differences of the wall potential for the case $\theta=0^{\circ}$ from those in Figs. 2-3 are worts noted. For $\theta=0^{\circ}$ there are no vertical currents in the core fluid along the magnetic field lines; as a result the top wall potential at $y^{\prime}=1$ coincides with the bottom wall potential at $y^{\prime}=-1$. Currents entering the $z^{\prime}=-1$ wall flow up the wall (for $y^{\prime}>0$ ) to the top and down (for $y^{\prime}<0$ ) to the bottom. The parabolic potential variation along that wall is symmetric about the $y^{\prime}=0$ midplane.

The fully developed pressure gradient is computed for various conductance ratios and magnetic angles. Figure 4 shows the pressure gradient, normalized by the value at $\theta=0^{\circ}$, for three duct aspect ratios, and $c=0.01$. The pressure gradient for $\theta=0^{\circ}$ is $\left[1+\frac{a}{c}+\frac{a^{2}}{3 c}\right]^{-1}$. The relative increase in pressure gradient is higher for higher aspect ratio or lower conductance ratio (not shown). It can be shown analytically that in a square duct the fully developed flow pressure gradient at $\theta=45^{\circ}$ is identical to that at $\theta=0^{\circ}$. In general, a small misalignment of the side wall relative to the magnetic field $\left(\theta<15^{\circ}\right)$, as would be expected in fusion blanket modules, always results in higher pressure drop than would be predicted if perfect alignment is assumed.

\section{Numerical Solutions in Fringing Magnetic Field}

The dimensionless axial variation of the magnetic field used in this study is shown in Fig. 5. For practical purposes, the tail of the $B_{y}(x)$ is smoothly leveled 
to a minimum value of 0.002 . Results presented in Figs. 6 and 7 are for a square duct using $c=0.01$ and $\theta=10^{\circ}$.

Figure 6 shows the dimensionless axial velocity profiles in the uniform field region where the flow is fully developed. In contrast to the results for the perfect field alignment, the core velocity is not everywhere uniform in both $z^{\prime}$ and $y^{\prime}$, with peaks occurring near the $z^{\prime}=-1$ wall. In the vicinity of the duct center, the velocity approaches 0.94 of the average velocity, compared to 0.76 for $\theta=0^{\circ}$ [1]. The qualitative behavior of the flow in Fig. 6 is expected because as $\theta$ increases from zero degree the high velocity jets in the thin side layers come out of the side layers and into the core. The velocity distributions in Fig. 6 are consistent with the fully developed flow results of Ref. 4.

Figure 7 shows the velocity profiles in the varying field region. Here threedimensional effects cause the velocity to increase near the side and decrease elsewhere, leaving a slowly moving region in the center. The increase in flow velocity near the side enhances the heat transfer characteristics there, such as the blanket first wall. However, the almost stagnant pocket of fluid in the center could have unfavorable heat transfer consequences.

Another key parameter of interest to designers of liquid metal blankets is the total MHD pressure drop. The total pressure is the sum of the fully developed pressure drop in the coolant channels and pressure drop due to three-dimensional effects. These 3D effects can arise trom manifolds, nonuniformity of magnetic field or duct cross section. Figure 8 shows the 3D pressure for a square duct with wall conductance ratio in the range of $0.002-0.2$. For $\theta=0^{\circ}, \Delta \mathrm{P}_{3 \mathrm{D}}$ increases linearly with $\mathrm{c}^{1 / 2}$ with a slope of 0.11 [1]. The value of $\Delta \mathrm{P}_{3 \mathrm{D}}$ is higher as $\theta>0^{\circ}$ and the linear dependence vanishes. 
Another approach, perhaps a more meaningful one, to assess the effect of $\triangle \mathrm{P}_{3 \mathrm{D}}$ in a fusion blanket is to determine the relative pressure increase over the computed fully developed pressure. A new variable is defined

$$
\ell_{3 D}=\frac{\Delta P_{3 D}}{\left.\frac{d p}{d x}\right|_{f . d .}}
$$

The three-dimensional length represents an added fictitious length to the coolant path over which the fully developed pressure drop is equivalent to the threedimensional pressure in the blanket system.

For the magnetic field distribution (Fig. 5) used in this analysis, the computed results of $\ell_{3 D}$ are shown in Fig. 9. For comparison, results for $\theta=0^{\circ}$ [1] are included. Since the coolant path length in a blanket is typically some tens of the characteristic length, the effect of the three-dimensional pressure is probably insignificant when $c>0.01$, but could adversely influence the design considerations if $\mathrm{c}$ is of the order $10^{-3}$ or less.

\section{Conclusions}

An inclined magnetic field in a rectangular duct causes (1) higher pressure drop, both fully developed and three-dimensional pressure, and (2) less flow concentration at the side walls than the case of parallel field. Both results could negatively impact the MHD/heat transfer performance of a liquid-metal-cooled fusion blanket.

Another key point of this study is the significance of the relative pressure increase due to three-dimensional effects in ducts with small wall conductance ratio (c $\leq 0.001$ ). This is important because the very reason for designing the blanket structure with a small wall conductance ratio is to control the MHD 
pressure below an allowable limit. In some designs, such as the breeding blanket for ITER [7], constraint in MHD pressure drop requires the duct walls to be electrically insulated from the liquid metal. Although the present analysis is not valid for insulated wall, the trend in Fig. 9 seems to indicate that as $\mathrm{c} \rightarrow 0$ the three-dimensional pressure can be comparable to or exceed the fully developed pressure drop in the main ccolant channels of the blanket.

\section{References}

[1] T.Q. Hua, J.S. Walker, B.F. Picologlou and C.B. Reed. Three-Dimensional Magnetohydrodynamic Flows in Rectangular Ducts of Liquid-Metal-Cooled Blankets. Fusion Technology 14 (1988) 1389.

[2] K.A. McCarthy, M.S. Tillack and M.A. Abdou. Analysis of Liquid Metal MHD Flow Using an Iterative Method to Solve the Core Flow Equations. Fusion Engineering and Design 8 (1989) 257.

[3] A. Ting, T.Q. Hua, J.S. Walker and B.F. Picologlou. Liquid Metal Flow in a Rectangular Duct with Thin Metal Walls and with a Non-uniform Magnetic Field. Int. Journal of Engineering Science 31 (1993) 357.

[4] L. Buhler. Liquid Metal Flow in Arbitrary Thin-Walled Channels under a Strong Transverse Variable Magnetic Field. Fusion Engineering and Design 17 (1991) 215.

[5] M. Tillack, A. Ying and H. Hashizume. The Effect of Magnetic Field Alignment on Heat Transfer in Liquid Metal Blanket Channels. University of California, Los Angeles Report, UCLA-FNT-024 (1989). 
[6] J.A. Shercliff. The Flow of Conducting Fluids in Circular Pipes under Transverse Magnetic Fields. J. Fluid Mech. 1 (1956) 644.

[7] T.Q. Hua and Y. Gohar. MHD Pressure Drops and Thermal Hydraulic Analysis for ITER Breeding Blanket Design. These proceedings. 


\section{Figure Captions}

Figure 1. Schematic of the duct cross section in dimensionless coordinates with an inclined magnetic field. The wall " $\mathrm{n}$ " has dimensionless length of 2.

Figure 2. Comparisons between numerical results of the top and bottom wall potential and the analytical solutions for a square duct, $c=0.01$ and $\theta=10^{\circ}$.

Figure 3. Comparisons between numerical results of the "side" wall potential and the analytical solutions for a square duct, $c=0.01$ and $\theta=10^{\circ}$.

Figure 4. Normalized fully developed pressure gradient for three aspect ratios and $\mathrm{c}=0.01$.

Figure 5. Dimensionless axial magnetic field distribution.

Figure 6. Axial velocity profiles in the uniform field region of a square duct with $c=0.01$ and $\theta=10^{\circ}$.

Figure 7. Axial velocity profiles in the varying field region of a square duct with $c=0.01$ and $\theta=10^{\circ}$

Figure 8. Three-dimensional pressure in a square duct with $c=0.01$. Results from Ref. 1 for $\theta=0^{\circ}$ are also included. 
Figure 9. Three-dimensional length derived from the three-dimensional pressure. Results from Ref. 1 for $\theta=0^{\circ}$ are also included. 


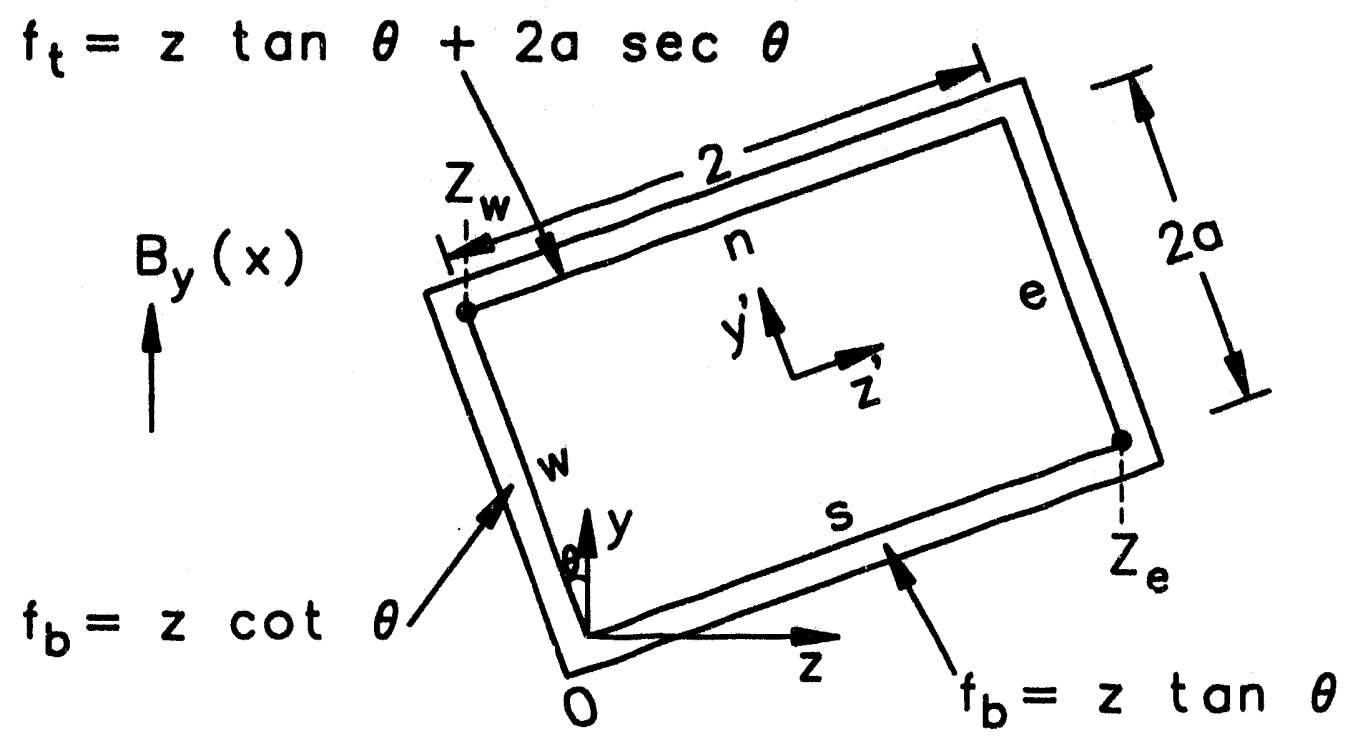

Figure 1

Thanh Q. Hua and John S. Walker

MHD Flow in Rectangular Ducts

with Inclined Non-Uniform

Transverse Magnetic Field 


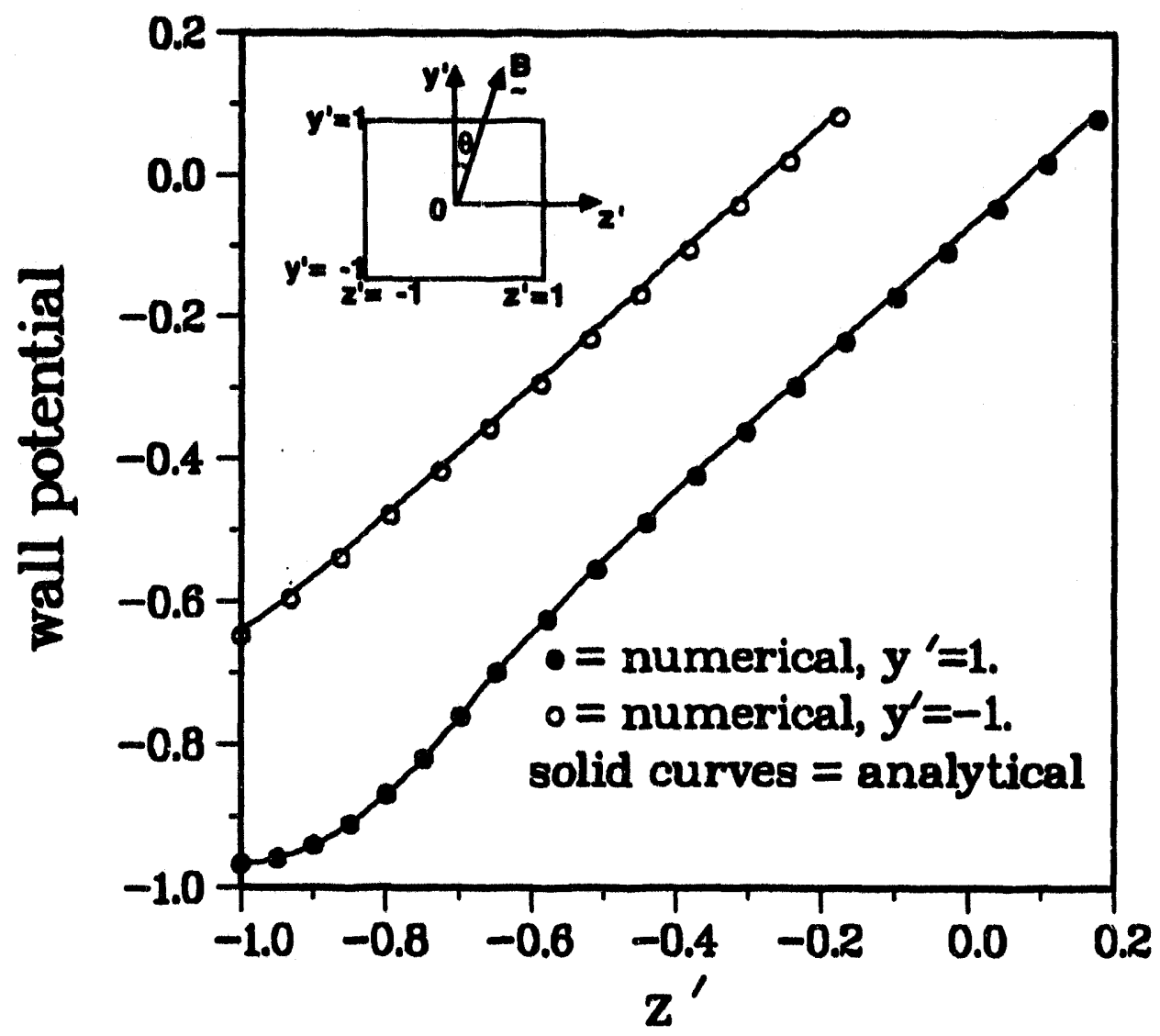

Figure 2

Thanh Q. Hua and John S. Walker

MHD Flow in Rectangular Ducts

with Inclined Non-Uniform

Transverse Magnetic Field 


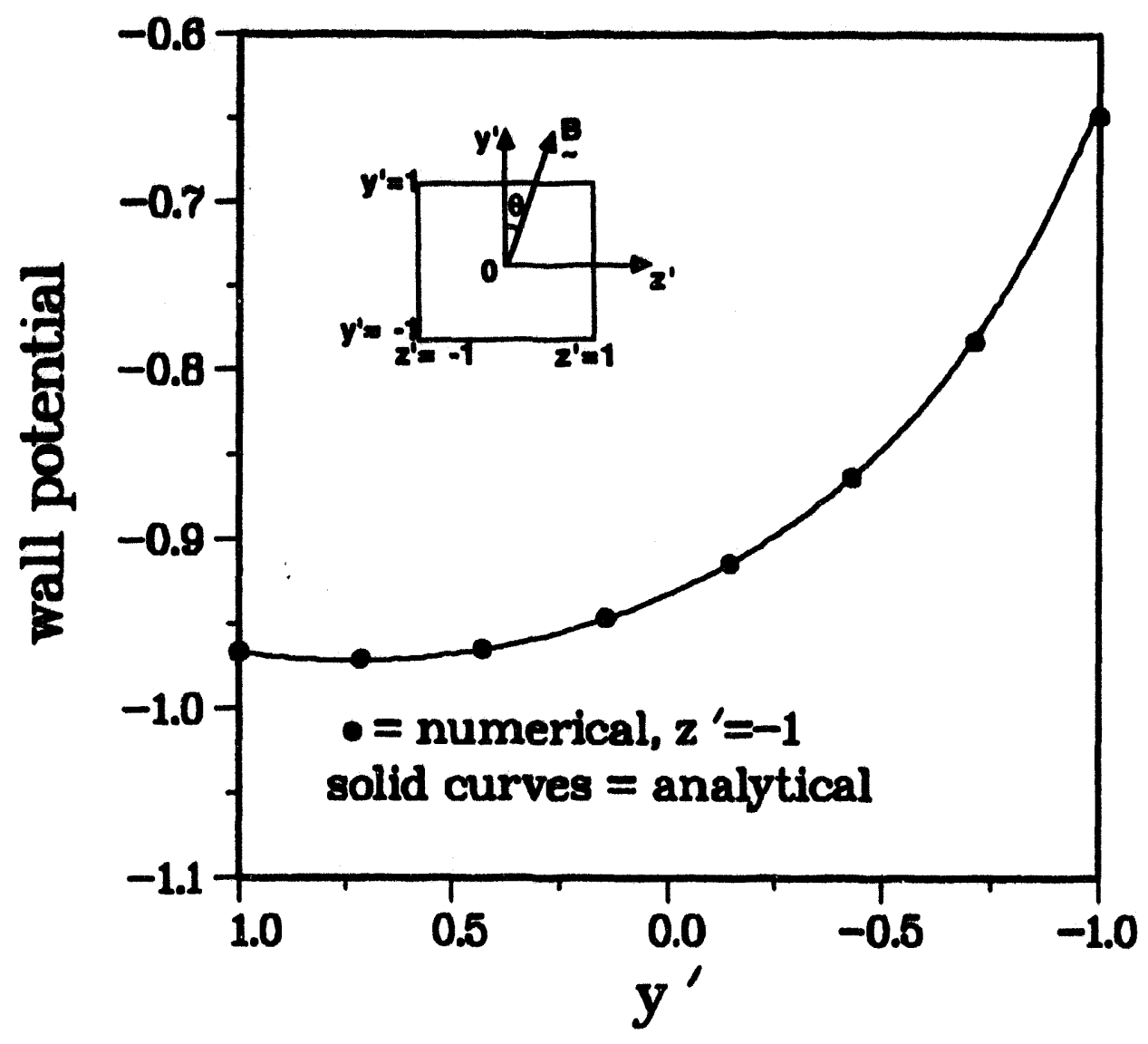

Figure 3

Thanh Q. Hua and John S. Walker

MHD Flow in Rectangular Ducts

with Inclined Non-Uniform

Transverse Magnetic Field 


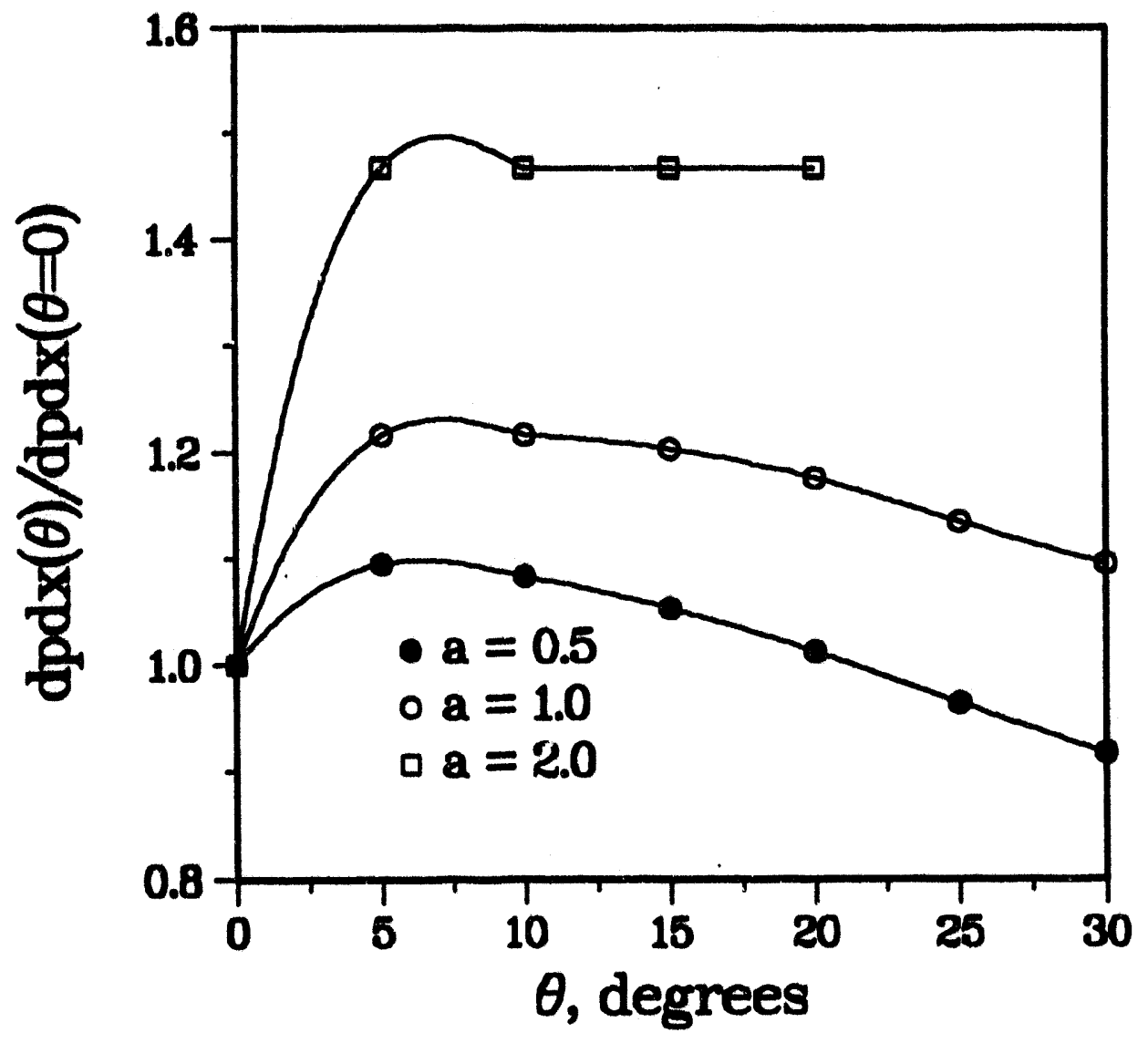

Figure 4

Thanh Q. Hua and John S. Walker

MHD Flow in Rectangular Ducts

with Inclined Non-Uniform

Transverse Magnetic Field 


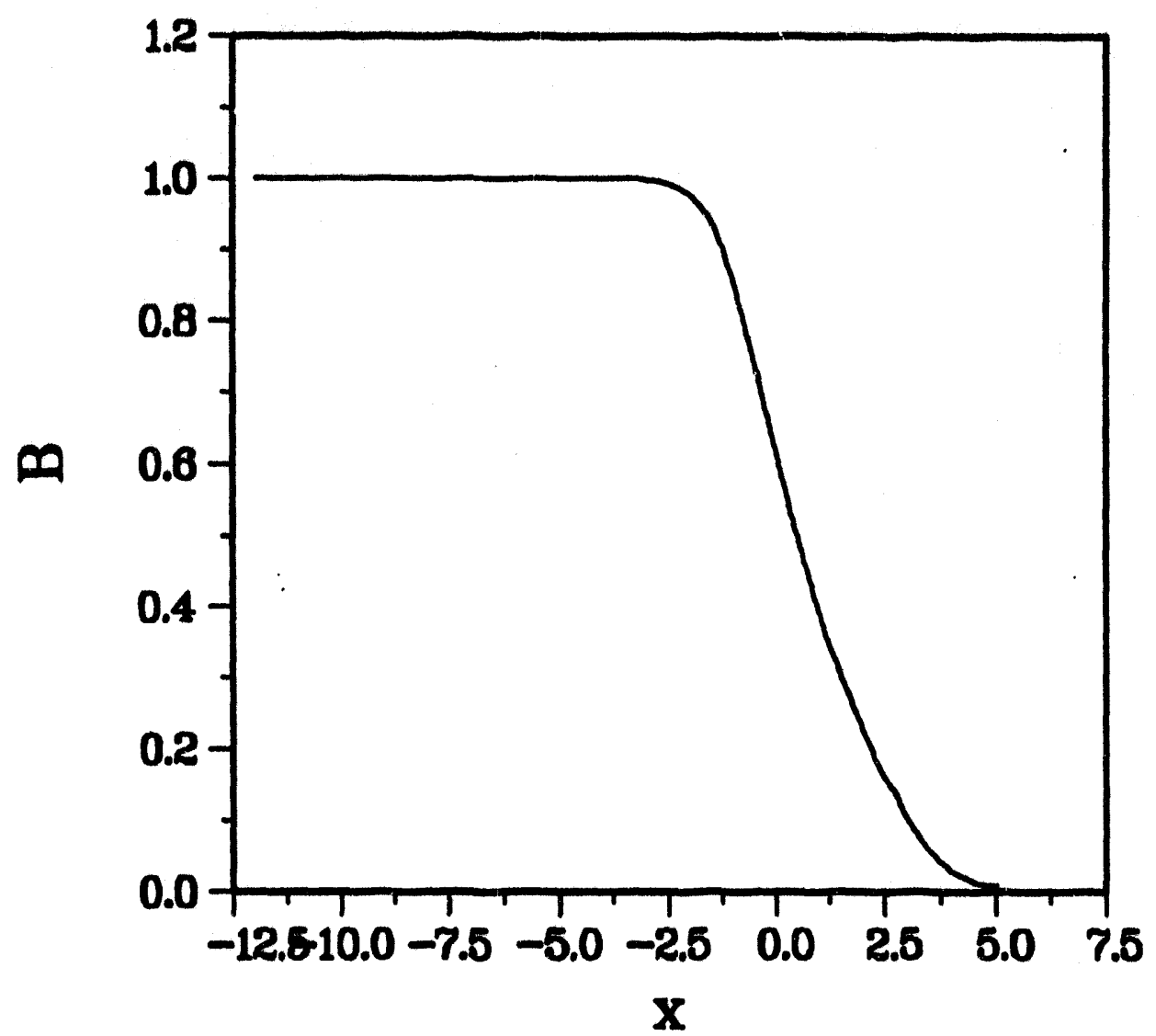

Figure 5

Thanh Q. Hua and John S. Walker

MHD Flow in Rectangular Ducts

with Inclined Non-Uniform

Transverse Magnetic Field 


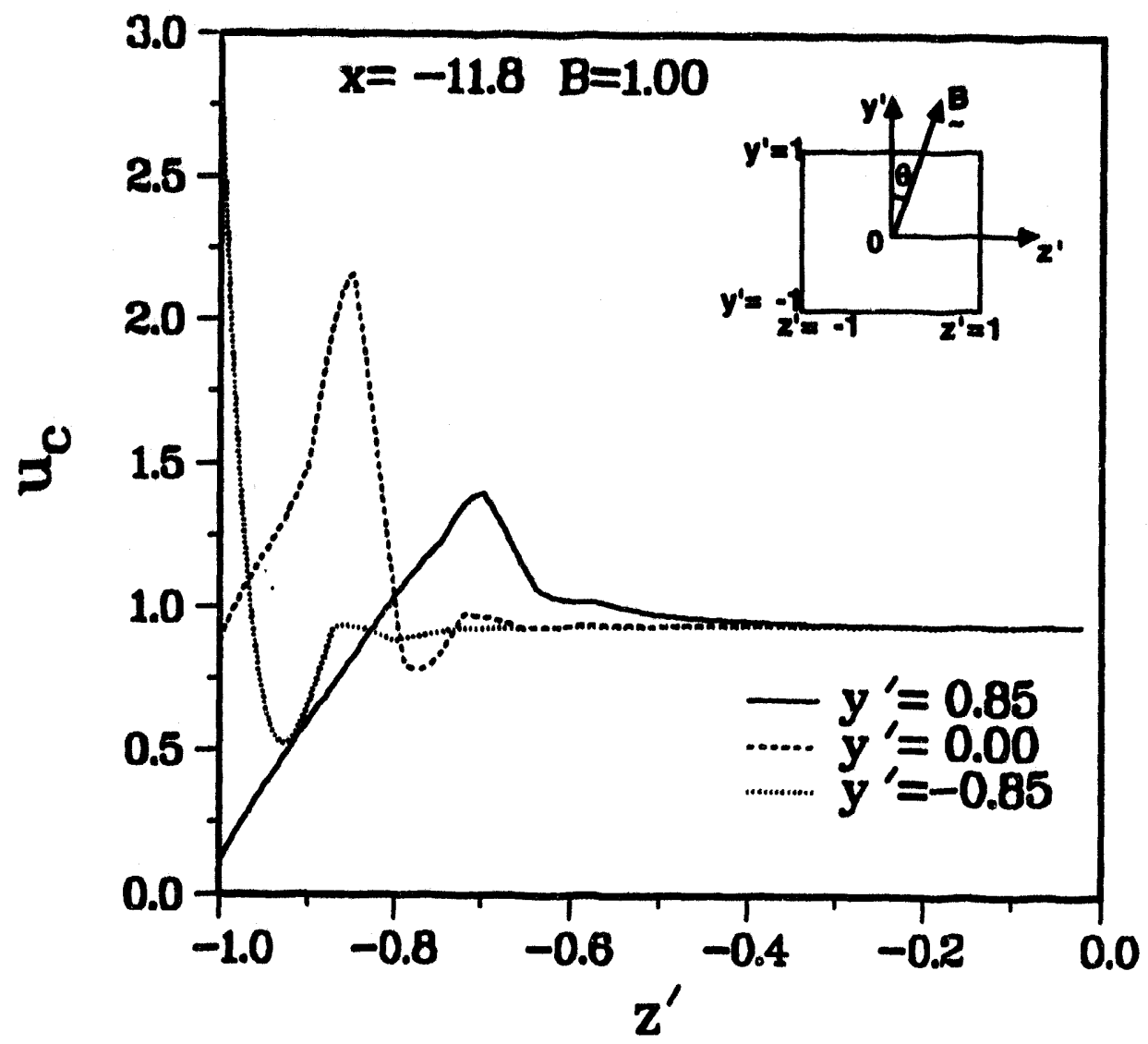

Figure 6

Thanh Q. Hua and John S. Walker

MHD Flow in Rectangular Ducts

with Inclined Non-Uniform

Transverse Magnetic Field 


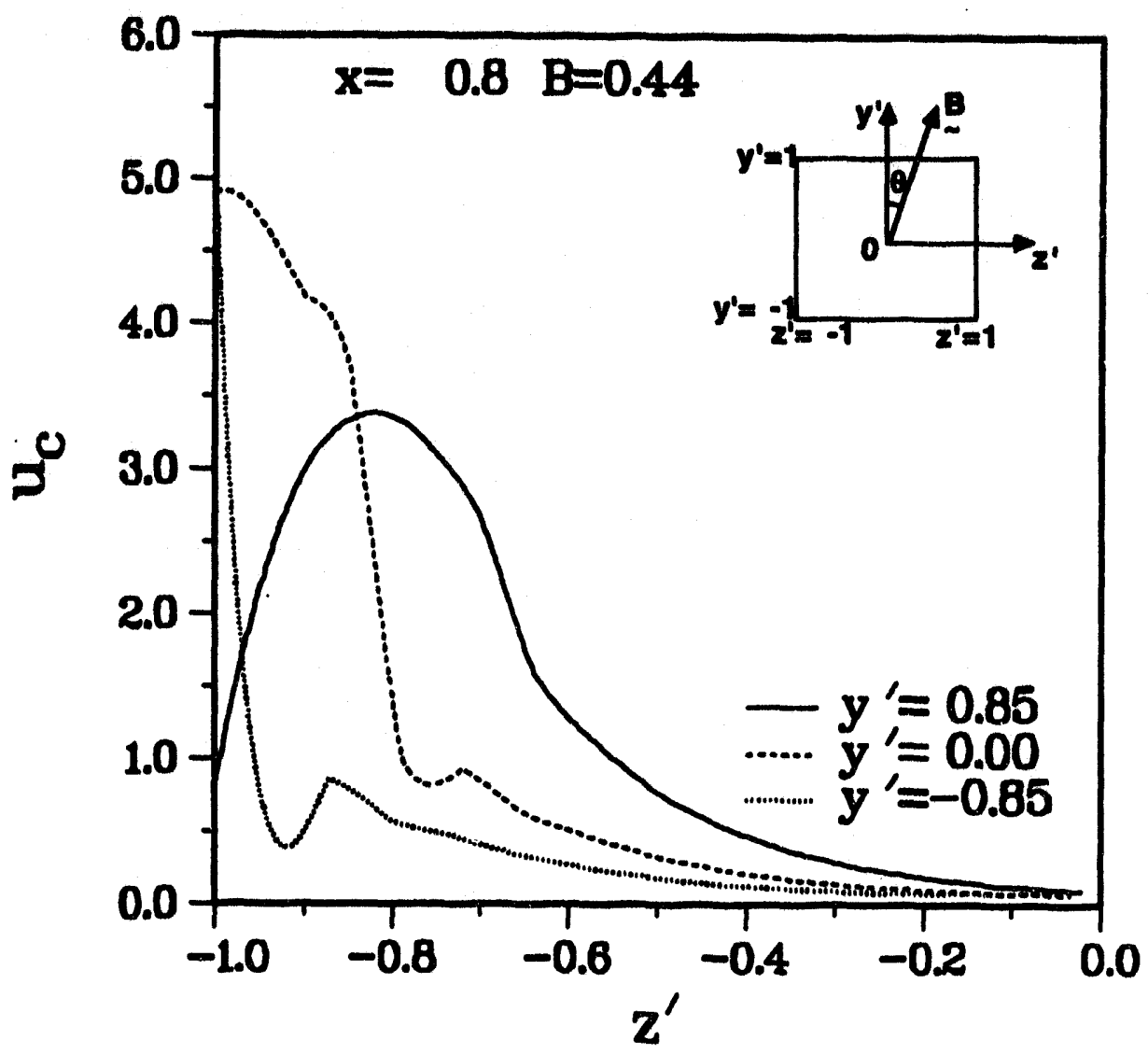

Figure 7

Thanh Q. Hua and John S. Walker

MHD Flow in Rectangular Ducts

with Inclined Non-Uniform

Transverse Magnetic Field 


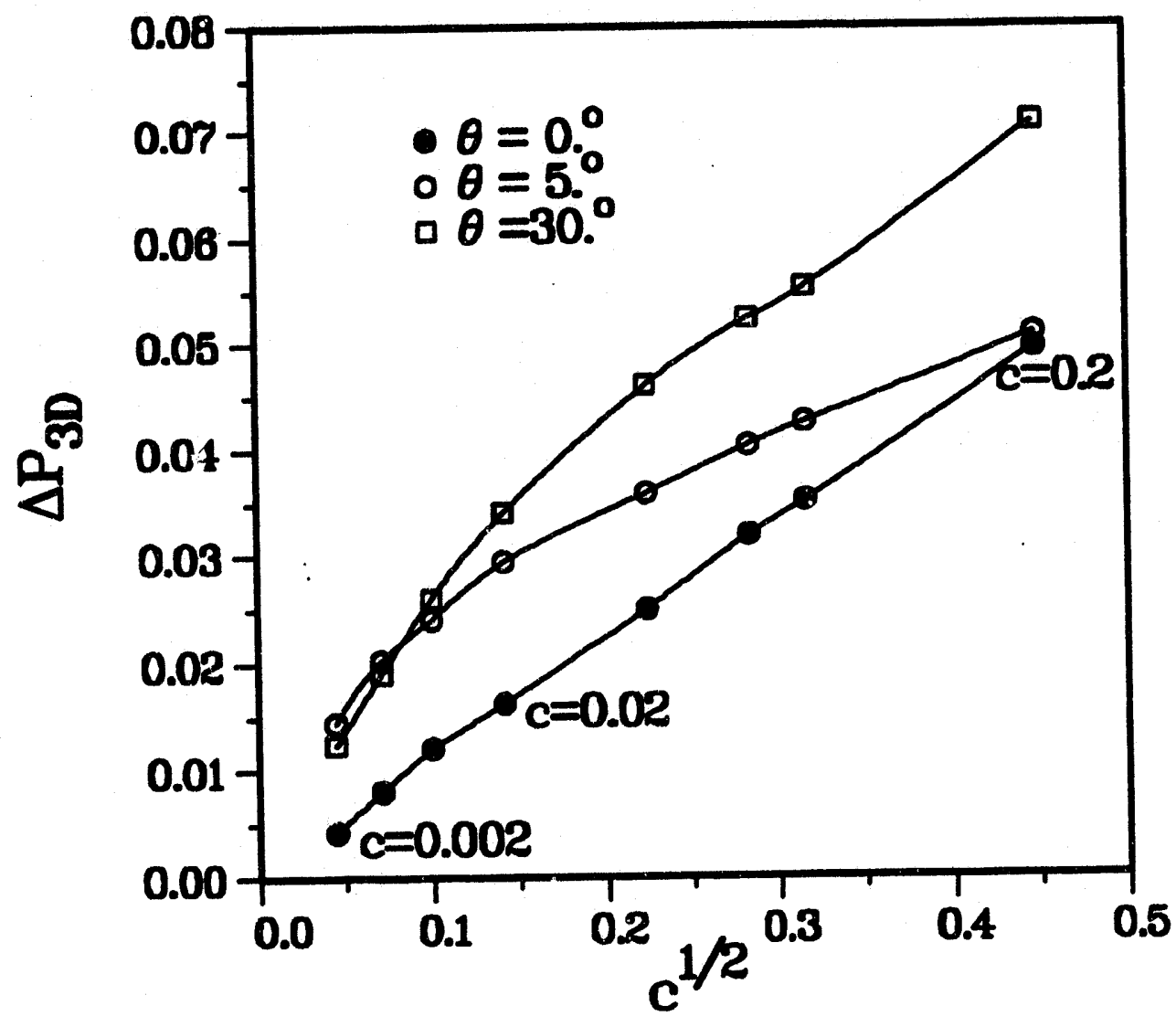

Figure 8

Thanh Q. Hua and John S. Walker MHD Flow in Rectangular Ducts

with Inclined Non-Uniform

Transverse Magnetic Field 

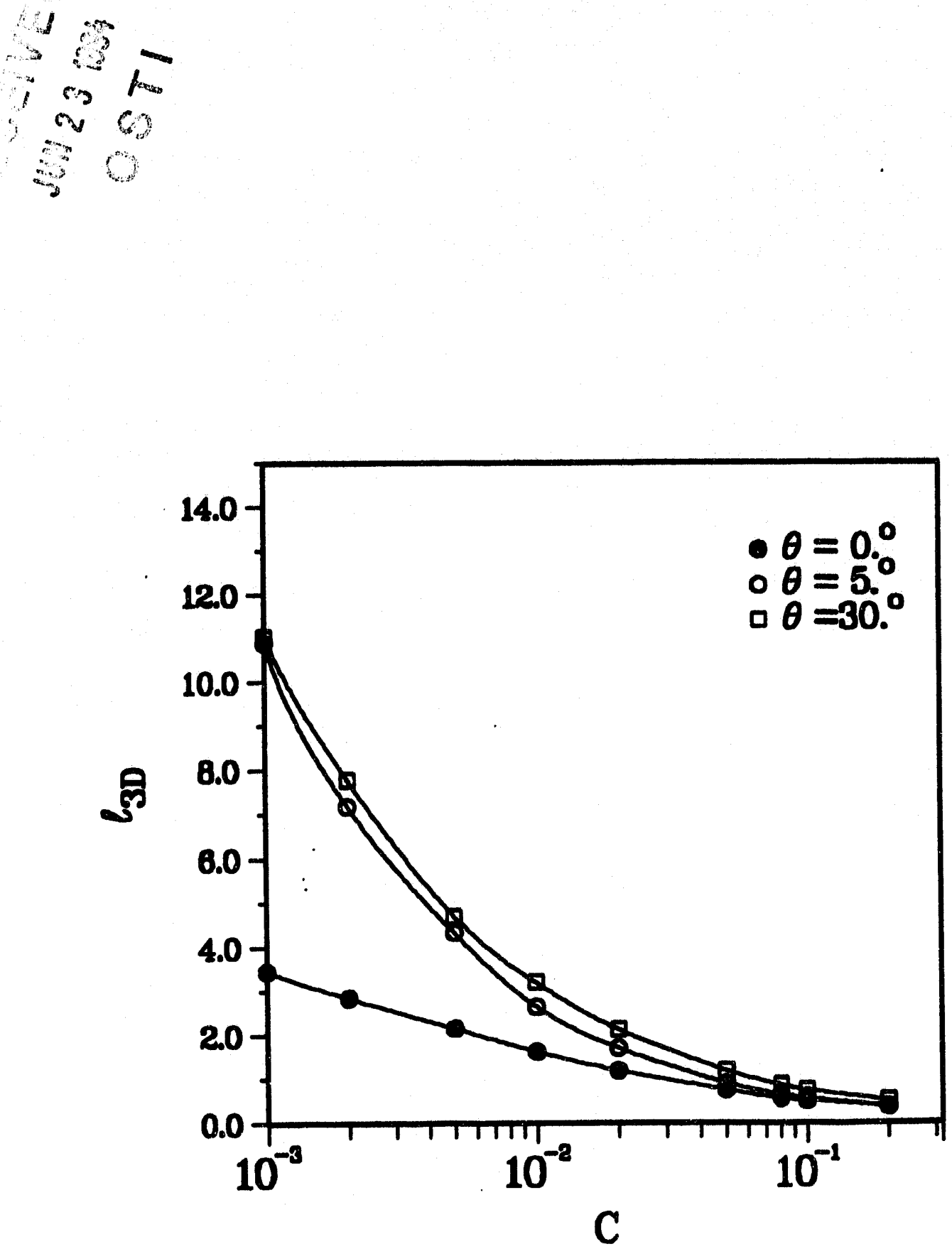

Figure 9

Thanh Q. Hua and John S. Walker MHD Flow in Rectangular Ducts with Inclined Non-Uniform Transverse Magnetic Field 

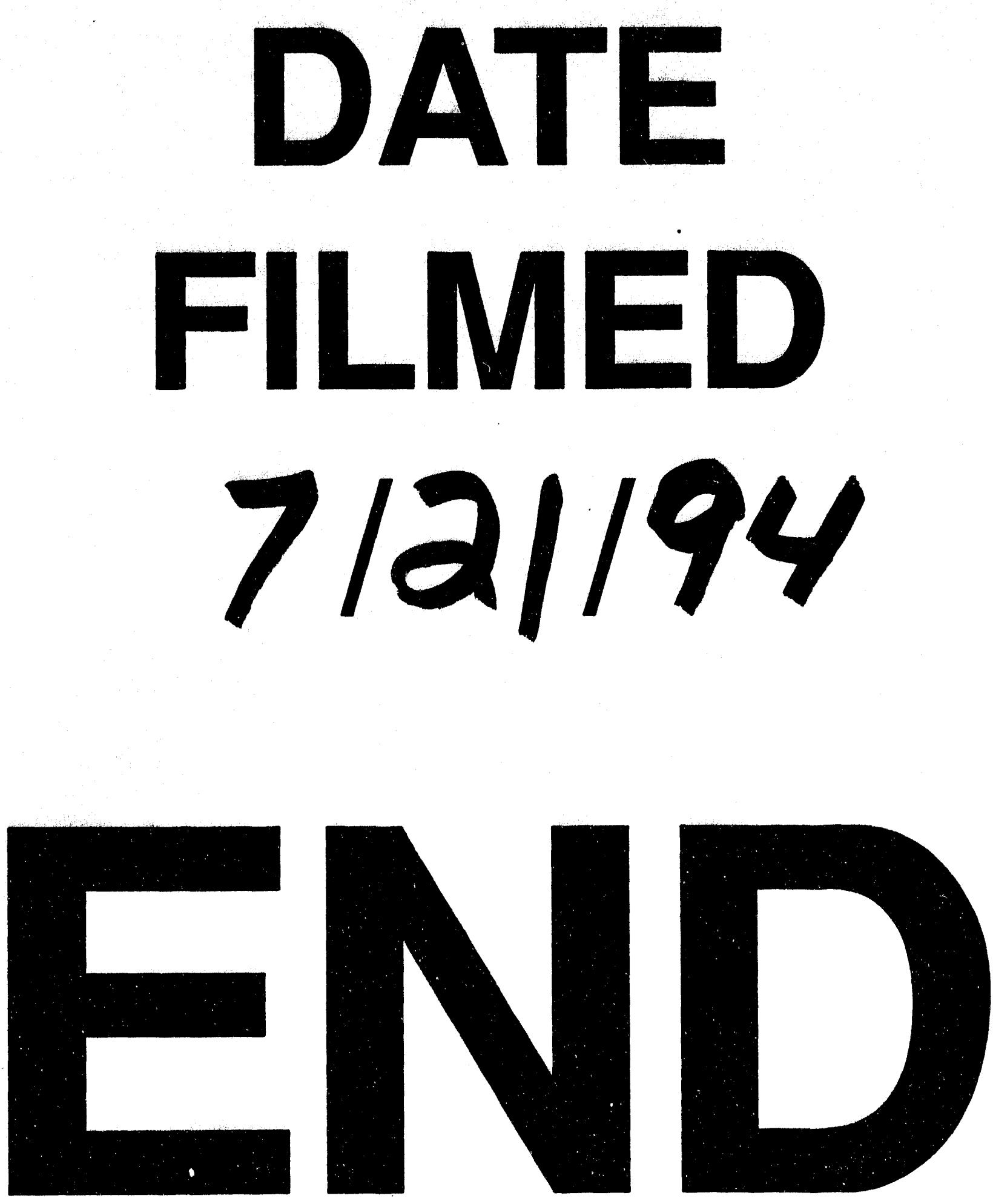
